\title{
Towards an European Approach for Characterisation of Multimaterial Micromanufacturing Process Capabilities
}

\author{
Markus Dickerhof ${ }^{1}$, Sabino Azcarate ${ }^{2}$, and Attila Temun ${ }^{3}$ \\ ${ }^{1}$ IAI, Karlsruhe Institute of Technology, Karlsruhe, DE \\ dickerhofakit.edu \\ ${ }^{2}$ Tekniker, Eibar, ES \\ sazcarate@tekniker.es \\ ${ }^{3}$ Production Engineering Dep., KTH-Royal Institute of Technology, SE \\ attilat@iip.kth.se
}

\begin{abstract}
The paper describes a comprehensive approach for the modelling and description of technologies available within a European Research Infrastructure consisting of distributed research infrastructures all over Europe. The resulting knowledge management system integrates the partial approaches of several European research activities in this area to a comprehensive description format for technical capabilities as well as maturity and economic aspects.
\end{abstract}

Keywords: Information and Knowledge management, Micro Manufacture, Nano Manufacture, Technological Interfaces, Process Ontologies, Technology Maturity.

\section{Introduction}

High multidisciplinary and low standardization are still key characteristics of emerging technologies such as MicroNano-Manufacturing (MNT). This observation has origin in ongoing significant technological developments regarding technical capabilities and process maturity. As a consequence stronger dependencies between design decisions and chosen manufacturing processes can be observed than e.g. macro mechanical engineering, where the dependencies between product requirements and technological capabilities in general much lower [1].

The fact that product development for NanoMicro-Systems requires expert knowledge from various areas such as sensor systems, biotechnology or electronic engineering increases the relevance of those dependencies and makes development much more challenging. When, maybe apparently despite the odds, a solution is reached, the acquired knowledge is highly dependent on the specific project and distributed across several data-mediums in heterogeneous formats, as such it is hard to reuse.

Consequently, possible promising ideas in Nano- Micro-Manufacturing and design are often discarded as lacking potential, because there is no way to access required 
knowledge in a centralized, homogeneous, shared, collaborative and cost-effective manner. It may well be the case that solutions to problems arising from these ideas have been in part already addressed in previous projects, however the lack of documented information renders these solutions inaccessible. Besides an incomplete documentation of the technical boundary conditions project reports and publications often solely refer to successful results and do not describe the failures and setbacks. An excellent tool to overcome these issues can be found using knowledge management methodologies and techniques.

This is the point where the MINAbase integrated Information and knowledge management approach, being further developed in the context of the European research infrastructure EUMINAfab [2] creates added value to the researchers work.

MINAbase is designed to collect, store and interpret information along the Microsystems development process in a way that allows the researchers of the participating European organisations and -projects and later also external users to benefit from the reuse of well structured data, collected in previous activities.

The paper describes the key modelling elements of the integrated EUMINAfab knowledge management system and gives an overview on the implementation status as well as an outlook to the activities, planned for the further filling of the database.

\section{EUMINAfab Information and Knowledge Management Framework}

The overall objectives to be achieved are:

- Provide a "solution neutral" but application specific interface between research groups when assessing user requests as well as along the prototyping or batch manufacturing.

- Provide a mapping of micro device functional aspects and manufacturing capabilities.

- Provide Design for Manufacture (DfM) generic rules that are EUMINAfab specific and open to the users of the infrastructure.

In the following we will explain how these objectives are addressed from the point of view. of a holistic process modelling.

\subsection{Description Basics and Technology Capability Maps}

It is important to stress that individual MNT are limited in their capabilities for producing 3D free-form micro structures in a wide range of materials. The way in which the key capabilities can be described was and still is a matter of discussion in many projects. In EUMINAfab seven categories of manufacturing processes have been classified, adapting and improving results such as from the European Network of excellence 4M. In EUMINAfab all technologies available within the consortium had been classified with respect to these seven categories. In addition a categorization scheme for characterization principles had been established (Figure 1). 


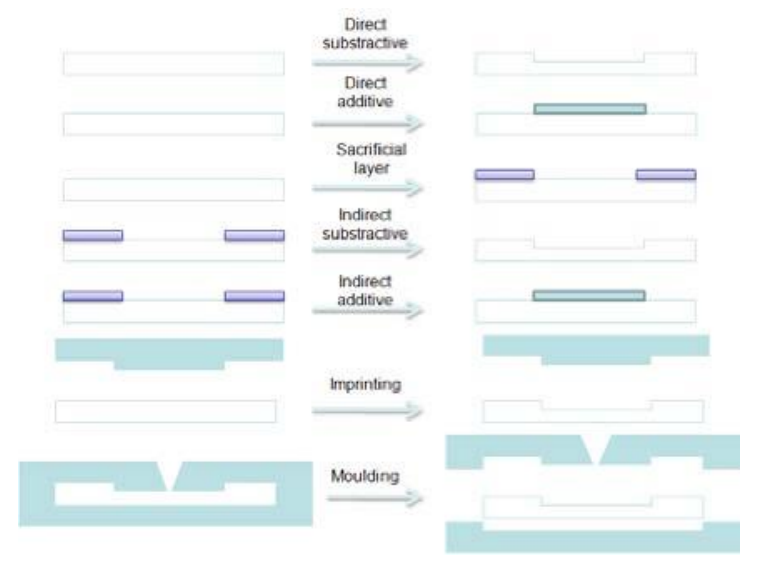

Fig. 1. Seven categories of Technologies [3]

In addition a scheme for description of technology related aspects has been introduced, providing information on of the following elements:

\section{Contact information}

Technological constraints (feature density, aspect ratio...)

Material classes and types (Polymers, ceramics,..)

Information about the technology (design rules, references,...)

Typical designs / structures (geom. Features, coatings, film,...)

Reference applications

Equipment (Machines, tools,...)

\subsection{Assessment of Technologies}

Assessment of technologies -allowing an oriented selection of appropriate manufacturing processes is essential for handling a user request. In practice, the maturity level of a given process is determined by characterizing it with a set of indicators that are representative of its overall behavior, performance and operational environment. The following indicators have been identified:

\section{Maturity Levels}

Maturity is "the state of being fully grown or developed" . From a manufacturing perspective, some of the characteristics of a mature process are that it is well understood, the necessary documentation and operator formal training are readily available and also it is continuously monitored and improved. Consequently, the performance and the overall behavior of such a process are highly predictable. Therefore, the maturity of a specific process or process pair provides one with the means to predict the likelihood of achieving particular process outcomes when it is used to fabricate a given product or component. Maturity levels are well-defined evolutionary stages towards achieving a mature Micro- Nano- manufacturing process 
or process pair. The following two description mechanism had been adopted from [4,5] for later implementation:

\section{Key Process Capability Areas}

The maturity of a given technology in regards to its key process capability areas (KPCA) can lie at any of the five levels described in Table 1. The KPCA maturity is a prerequisite for achieving or improving the overall maturity of a process. The maturity indicators can be used in determining whether different issues and goals related to the process capability parameters and their associated KPCA have been addressed or whether their key parameters metrics have been improved.

Table 1. Process Capability Maturity Levels

\begin{tabular}{|c|c|c|}
\hline \multicolumn{2}{|l|}{ Levels } & Description \\
\hline Initial & 1 & $\begin{array}{l}\text { Introduction of a new process. Undocumented } \\
\text { and dynamically changing. Initial (chaotic, ad } \\
\text { hoc) utilization of a new process. }\end{array}$ \\
\hline Repeatable & 2 & $\begin{array}{l}\text { A process with a predictable behaviour. } \\
\text { Consistent and repeatable results are } \\
\text { achievable if rigorous discipline is applied. } \\
\text { The process is used repeatedly with } \\
\text { predictable results. }\end{array}$ \\
\hline Defined & 3 & $\begin{array}{l}\text { Standard Process. Subject to improvements. } \\
\text { Defined (institutionalized) process. A process } \\
\text { approved for given applications or product } \\
\text { requirements. }\end{array}$ \\
\hline Managed & 4 & $\begin{array}{llr}\text { Validated process with a broad usage. } \\
\text { Adaptable to given needs/requirements. } \\
\text { Validated process capabilities. Quantified } \\
\text { process management and established } \\
\text { measurement practices. }\end{array}$ \\
\hline Optimized & 5 & $\begin{array}{l}\text { Process with high predictability and } \\
\text { performance. Incremental innovative changes. } \\
\text { Defined improvement objectives. Optimized } \\
\text { management practices. Planned and well } \\
\text { managed process optimization/ improve- } \\
\text { ments. }\end{array}$ \\
\hline
\end{tabular}

\section{Process Maturity Indicators}

These indicators describe the typical benchmarking activities, characteristics and performance metrics for each maturity level. For example, an indicator for the maturity of the "Part Size \& Complexity", "Material" and "Processing" KPCA could be the existence of a correlation between analytical modeling/simulation results and actual process performance in a given environment.

To implement the proposed methodology it is necessary to identify a set of maturity indicators, e.g. through Delphi-type workshops with MNT process experts. The proposed modeling approach was applied to assess the maturity of different stateof-the-art micro nano- manufacturing processes integrated within EUMINAfab.

\subsection{Technical Aspects for Description of Technical Aspects}

To ensure a reuse of process related information the methodology should, besides the maturity related parameters, allow for an order-independent description and storage of 
technology-oriented parameters. The ProWiDa methodology for classifying aspects of influences through taxonomies with inheritance bases on the assumption that for an adequate description of technological capabilities all influencing aspects (technical, economic, human, etc.) need to be considered [6].

For the technical section the following "aspects" have been identified as of relevance:

- Materials (substrates and layers)

- Procedure

- Most critical geometry

- Machines

- Tools

\subsection{Integration of All Aspects of Influence on "Competence" Level}

Competences represent at first hand a n-tuple of the above mentioned aspects (see Figure 2).

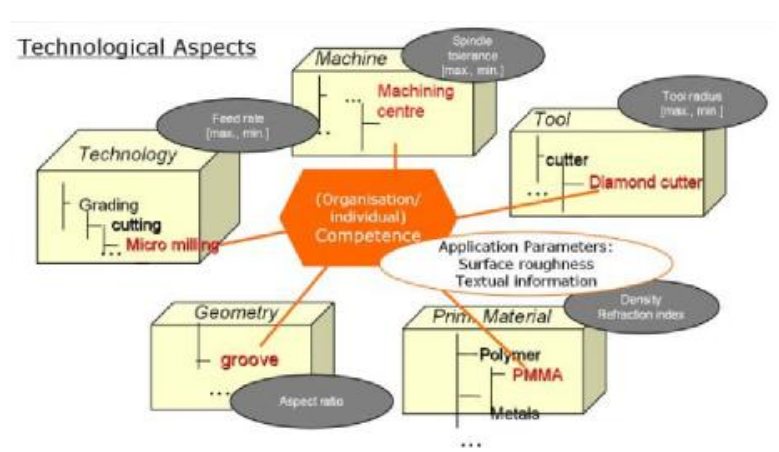

Fig. 2. Technology-oriented "aspects" of a process step

Competences may contain -in addition to the underlying aspect-related parameterson "compe-tence level" additional parameter sets representing the technical capabilities of an organisaton unit (Figure 3).

- Process parameters describe typical technical factors that can be achieved in a reliable manner, such as width, length, material parameters, milling tool size, feed, milling velocity, etc. Also economical parameters such as machine costs can be attached at this level.

- Application parameters describe the resulting capabilities being aimed for, e.g. resulting minimum groove width, surface roughness, which again correlate with application specific requirements coming from physical effects.

- Design rules are used to describe expert knowledge that cannot be reduced to a parameter level (e.g. design rules of a mold)

- Besides specific application related technical parameters, maturity related and economic aspects can also be placed on competence level since they usually correspond not only to a specific technology but also to the material chosen, etc. 
In addition these combinations (competences) usually are dedicated to a specific group or company.

\begin{tabular}{|c|c|}
\hline \multirow{3}{*}{$\begin{array}{l}\text { Competence } \\
\text { parameter }\end{array}$} & $\begin{array}{l}\text { Cross-competence parameter values } \\
(\text { e.g. accuracy, TRL, costs ...) }\end{array}$ \\
\hline & $\begin{array}{c}\text { Competence (Class) specific parameter values } \\
\text { (e.g. surface roughness ...) }\end{array}$ \\
\hline & $\begin{array}{c}\text { Unstructured competence-related information } \\
\text { (e.g. pictures, graphs, prosa ...) }\end{array}$ \\
\hline \multirow{4}{*}{$\begin{array}{c}\text { Competence } \\
\text { - related } \\
\text { Aspects }\end{array}$} & $\begin{array}{l}\text { related MACHINES Aspect specifc parameters } \\
\text { (e.g. machine workspace parameters) }\end{array}$ \\
\hline & $\begin{array}{l}\text { related TOOLS Aspect specifc parameters } \\
\text { (e.g. Tool size) }\end{array}$ \\
\hline & $\begin{array}{c}\text { Generic MATERIAL Aspect specifc parameters } \\
\text { (e.g. viscosity, Youngs modulus,...) }\end{array}$ \\
\hline & Basic Feature aspects (angle, dimension, ...) \\
\hline
\end{tabular}

Fig. 3. Installation, technology and aspect specific Parameter sets in a competence

Instantiations of these competences can be used to store information gained along the processing of a user request. The specific combination of aspects is the called "process step" and represents the smallest unit of a process chain described in the next chapter in a more detail.

Through its full database representation the description methodology again allows for an automated generation of installation-, material, technology and feature-specific datasheets on a very detailed description level.

\subsection{Modelling of Process Chains}

In the ProWiDa methodology three major elements allow for the description of a complete production process (Figure 4).

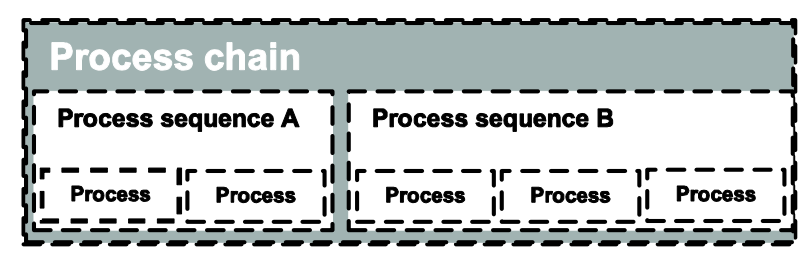

Fig. 4. Scheme of the major modelling elements in MINAbase according to the Matroshka Principle [7]

- The process chain represents a more or less complete order / product specific set of process steps. Hence the resulting parameters on this highest aggregation level are of relevance for the application.

- Process sequences represent a characteristic of a basic technology. The technology is represented by a set of process steps. 
- The process step itself builds the smallest modelling element, which represents a subtask that has to be executed when processing a technology.

An example sequence for such a generic microsystems related process sequence is shown in Figure 6.

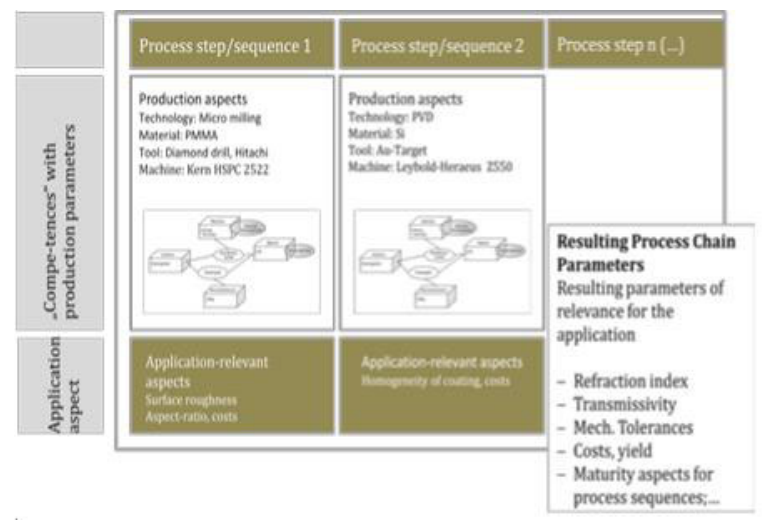

Fig. 5. Process sequences in the ProWiDa Methodology

In EUMINAfab an implementation of a specific realisation of processes sequences based on the idea of using pairs in order satisfy specific application requirements will be implemented (see Figure 6). In this case the pre and post conditions of the ProWiDa methodology are equivalent to the technological interfaces.

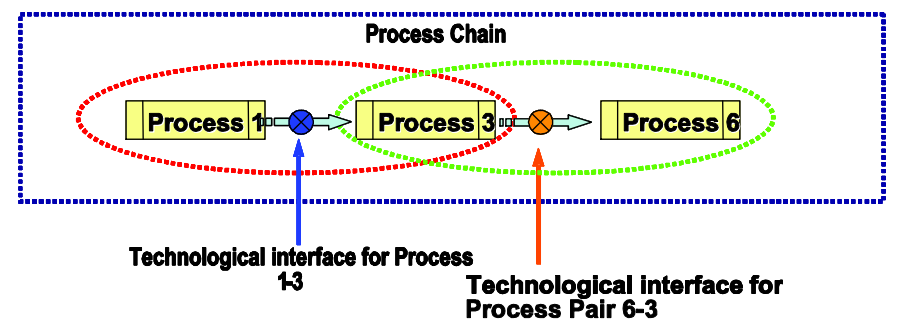

Fig. 6. Process Pairs and Process Pair Technological Interfaces [8]

\section{Implementation and Filling of the Database}

It is obvious to mention, that methodologies are only as good as their acceptance in the end user community. "Acceptance" means -besides the acceptance of the methodology itself- to ensure, that the users are set into the position to handle underlying procedures and data in an as intuitive as possible manner. In EUMINAfab two systems had been developed/adapted and set up: 


\subsection{Information Management System}

The Information management system has three key functions in EUMINAfab

- It supports the operational activities of the network management through an implemented semi collaborative workflow engine [9]. The engines allow for a user request-oriented provision of information and tools along the processing of a user request. This feature gains importance with an increasing amount of tasks being processed in parallel.

- A set of internal organisational processes for intake, evaluation, work breakdown, realisation as well as procedures to escalations had been defined to ensure quality and repeatability of decisions.

- Information systems also ensures a well structured collection of manufacturing related data in a traceable way, which is mandatory for a later analysis of decision processes regarding technological and economic relations.

\subsection{Implementation in the MINAbase Online-Database}

Since the MINAbase concept is based on the collection of hundreds of individual competences, a complex ontology has been built up, allowing for a highly flexible storage of technological competences.

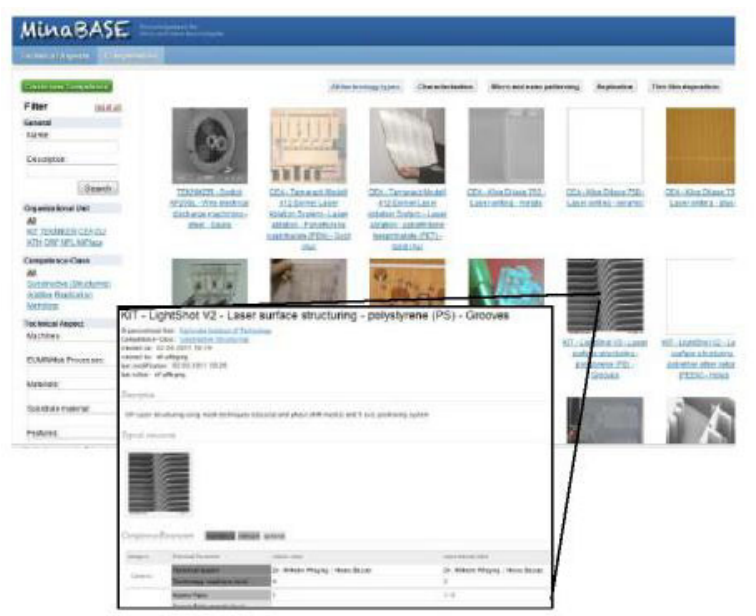

Fig. 7. MINAbase retrieval page with EUMINAfab Competences

The retrieval of data in the different taxonomies of the MINAbase ontology is ensured through full text search functionality as well as through the implementation of filtering mechanisms (Figure 7). For the remaining time of the EUMINAfab project it is planned to expand this toolset by case based reasoning strategies for an improved retrieval and also by a modelling module to allow for the modelling of constraints between process steps in a micro manufacturing process chain. 


\subsection{Filling the Database}

Initial Datasets have been collected at the very beginning of the EUMINAfab project in the first version of the technology capability maps. However the ability to assess the appropriateness of a technology regarding a given user task requires an in depth knowledge on the technological capabilities according to the description methodology mentioned above.

\section{Incorporation of benchmarking results}

Benchmarking is used in EUMINAfab for ranking purposes, and therefore it could eventually also be applied for process chain selection when there are alternative competing solutions for the fabrication of a given micro component.

In MINAbase the production and analysis of special artefacts has been established to collect information on the process capabilities and especially on the quality constraints. A number of artefacts have been machined in JRA3.1 and will in early 2011 be analyzed by KTH using high performance metrology systems. [10]. As an example, in the accomplished measurements we have found cases where machine accuracy was off by an order of magnitude.

Deviations of such large extent definitely call for corrective actions to improve the quality of EUMINAfab installations. Laboratory audits have been performed by NPL personal at different installations with both good and bad results regarding traceability issues. An inventory of machine-to-machine substrate handling has been performed.

It is the responsibility of each partner to update the installation performance in MINAbase. Still it is striking that universities can put lots of investment in machining and characterization equipment but hesitate to buy traceable test artefacts for a fraction of the equipment cost. Notwithstanding in the last few months the interest of the different manufacturing units to perform machining tests just to evaluate the performance of their equipment has been strengthened.

\section{Results from first stage quality assessment}

The establishment of a quality management system to collect parameters from user requests is getting close to being fulfilled. By evaluating the outcome of a certain number of user projects each year we can also identify and evaluate the selection principles used so far.

Moreover the EUMINAfab knowledge database will benefit from each user project. As an example of necessary feedback, the discrepancy between experts predicted time to fulfill a process task and the actual time needed to do it differs significantly at several installations. With that experience brought into the knowledge database the likelihood of better planning is obvious. A drawback of the relatively few fulfilled projects is the large spread among the process requests, however.

\section{Outlook}

The methodology presented in this paper is actually lacking of two important aspects:

Application related aspects are only considered indirectly, e.g. by the provision of information about geometrical aspect or the maturity related aspects. To ensure the usability of the MINAbase approach aspects such as the specifc requirements of 
process scalability in the biosector, relevance of technologies for a certain industry need to be also collected. Secondly aspects being of relevance for the later assignment of technologies to a process chains and related procedural rules need to be implemented to ensure compatibility between consecutive process steps. Indirectly related to this is also the pending connection to design tools for modelling of physical behaviour of micro enabled parts.

\section{References}

[1] Dickerhof, M.: A Cooperation Model and Demand-Oriented ICT Infrastructure for SME Development and Production Networks in the Field of Microsystem Technology. In: Ratchev, S. (ed.) IPAS 2010. IFIP AICT, vol. 315, pp. 319-328. Springer, Heidelberg (2010) ISBN 978-3-642-11597-4

[2] Kautt, M., Anson, S.M., Saile, V., Scholz, S., Fugier, P., Lambertini, V., Abad, E., Dirne, F., Loeschner, H., Leach, R.K.: Facilitating Open Innovation in Micro and Nano Technology by Providing Open Access to a Pan-European Toolbox called EUMINAfab. In: Proceedings of the 4M/ICOMM 2009 - The Global Conference on Micro Manufacture, pp. 11-24 (2009)

[3] Azcarate, S., Uriarte, L., Schoth, A., Bigot, S., Tossello, G., Roth, S., Staemmler, L.: Hybrid Tooling: A Review of Process Chains for Tooling Microfabrication in 4M. In: Proceedings of the 4M 2006 - Second International Conference on Multi-Material Micro Manufacture, pp. 305-308 (2006)

[4] Brousseau, E.B., Barton, R., Dimov, S.S., Bigot, S.: Technology Maturity Assessment of Micro and Nano Manufacturing Processes. In: Proceedings of the 4M/ICOMM 2009 The Global Conference on Micro Manufacture, Karlsruhe (2009)

[5] Fraser, P., Moultrie, J., Gregory, M.: The Use of Maturity Models/ Grids as a Tool in Assessing Product Development Capability. In: Proceedings of the IEEE International Engineering Managmenent Conference, IEMC 2002 Conference, pp. 244-249 (2002)

[6] Dickerhof, M., Parusel, A., Langbein, I.: Increasing efficiency and transparency of micro system product and process modeling - from process documentation to process knowledge management in micro systems technology. In: Teti, R. (ed.) Proc. of the 5th CIRP Internat. Seminar on Intelligent Computation in Manufacturing Engineering (CIRP ICME 2006), Ischia, I, July 25-28, pp. S563-S567 (2006) ISBN 88-95028- 01-5

[7] Parusel, A., Dickerhof, M., Binnig, C., Gengenbach, U.: Doing the next step - from a process related documentation to process knowledge management in non silicon Micro Systems Technology. In: Proc. of the 9th Internat. Conf. on the Commerzialization of Micro and Nano Systems (COMS 2004), Edmonton, CDN, August 29-September 2, pp. S115-S122. MANCEF, Albuquerque (2004)

[8] Vella, P.C., Brousseau, E.B., Minev, R., Dimov, S.S.: A Methodology for Technology Maturity Assessment of Micro and Nano Manufacturing Processes and Process Chains. In: Proceedings of the 4M/ICOMM 2010 - The Global Conference on Micro Manufacture, Oyonnax (2010)

[9] Dickerhof, M.: INFOFLOW - a process oriented workflow and information system for micro system development and production (INIWA 2002). In: 35th Hawaii Internat. Conf. on System Sciences, Hilton Wakikoloa Village, Hawaii, January 7-10 (2002)

[10] Ekberg, P., Stiblert, L., Mattsson, L.: Ultra-precision geometrical measurement technique based on a statistical random phase clock combined with acoustic-optical deflection. Meas. Sci. Technol. 21(125103) (2010) 